\title{
La educación afectivo-sexual en el sistema educativo español: análisis normativo y posibilidades de investigación
}

\author{
Mayte BEJARANO FRANCO \\ Antonio MATEOS JIMÉNEZ ${ }^{2}$
}

\section{Introducción}

La principal finalidad de este artículo es llevar a cabo una revisión en materia de legislación educativa sobre la importancia que se le otorga a la educación afectivo-sexual (EAS) en las leyes educativas españolas desde 1990 hasta la actualidad. Se trabaja este aspecto legislativo entendiendo que educar en materia afectivo-sexual es algo realmente prioritario que debe estar prescrito en las etapas de Educación Infantil y Primaria así como en la formación del profesorado. Esto último queda recogido en la Ley Orgánica 1/2004 de 28 de diciembre, de Medidas de Protección Integral contra la Violencia de Género (ESPAÑA, 2004). Esta ley señala en su artículo 7, con respecto a la formación del profesorado, la necesidad de que se incluya la educación para la igualdad entre hombres y mujeres en la formación inicial y permanente de los docentes para que así adquieran las habilidades necesarias centradas en:

Educar "en el respeto de los derechos y libertades fundamentales y de la igualdad entre hombres y mujeres y en el ejercicio de la tolerancia y de la

1 UCLM - Universidad de Castilla la Mancha. Campus Ciudad Real - Departamento de Pedagogía. Ciudad Real - Espańa. 13071 - mariateresa.bejarano@uclm.es

2 UCLM - Universidad de Castilla la Mancha. Campus Toledo - Departamento de Pedagogía. Toledo - Espańa. 45071 - antonio.mateos@uclm.es 
La educación afectivo-sexual en el sistema educativo español: análisis normativo y posibilidades de investigación

libertad dentro de los principios democráticos y de convivencia”, prevenir conflictos y resolverlos de manera pacífica "en todos los ámbitos de la vida personal, familiar y social" y fomentar "actitudes encaminadas en el ejercicio de iguales derechos y obligaciones por parte de mujeres y hombres tanto en el ámbito público como privado y la corresponsabilidad entre los mismos en el ámbito doméstico".

La ley también trata aspectos relacionados con la educación en la diversidad afectivo-sexual ya que la violencia de género y la violencia hacia las personas está directamente relacionada e implica aludir a la violencia que se ejerce contra las libertades sexuales (PENNA, 2012).

Asimismo, nos parece interesante plantear la importancia de que el maestro y maestra asuman, dentro de su profesión, la investigación como una función reflexiva y proactiva (BEJARANO; RODRIGUEZ TORRES, 2015) dada la naturaleza de dicha profesión. El contacto educativo con personas hace necesaria la indagación y tratamiento de todas las vertientes humanas, considerando además que la atención a la sexualidad es un derecho y merece conocimiento exhaustivo que debe ser tratado en el ámbito educativo desde edades tempranas (CEVALLOS, 2014; MALÓN, 2012)

Una buena y, sobre todo, temprana educación afectivo-sexual en las etapas educativas puede ayudar a suprimir roles, estereotipos y actitudes de corte racista, homófoba y xenófoba con el fin de prevenir y evitar discriminaciones, malos comportamientos en relación al sexo y/o al género, etnia, cultura, religión o ideología. El tratamiento educativo integral de lo afectivo y sexual amplía la perspectiva educativa hacia nuevas realidades sociales que son preciso abordar desde la consideración beneficiosa y enriquecedora que merecen. En el ámbito educativo, se debe insistir en el hecho de que todas las opciones sexuales pueden coexistir, basándonos en el principio fundamental de igualdad.

El objetivo que se plantea en el presente artículo es doble. Por una parte, dejar constancia de cómo se ha venido tratando la EAS en el sistema educativo español desde 1990 y, en segundo lugar, hacer una aproximación, desde un enfoque investigador, para abordar tales contenidos en el ámbito de la formación de docentes.

Para ocuparnos de la parte legislativa, llevaremos a cabo una lectura reflexiva a partir de un proceso indagatorio sobre los preámbulos ${ }^{3}$ de cuatro leyes educativas españolas, concretamente: LOGSE (ESPAÑA, 1990); LOCE (ESPAÑA, 2002); LOE (ESPAÑA, 2006) y LOMCE (ESPAÑA, 2013).

3 Se han revisado los preámbulos de las leyes pues en ellos, es donde se tratan las intenciones educativas del sistema educativo de un país. 


\section{La educación afectivo-sexual en las leyes españolas: desde la LOGSE hasta la LOMCE}

Con el fin de acercarnos al significado de la educación afectivo-sexual y llevar a cabo el proceso indagatorio legislativo, partimos de la definición sobre el término que realizan algunas instituciones y autores/as especialistas. La Oficina Regional para Europa de la Organización Mundial de la Salud y la organización alemana BZgA (Centro Federal de Educación para la Salud) define el término como:

La educación sexual es también parte de una educación más general y, por lo tanto, afecta el desarrollo de la personalidad de niñas y niños. Su naturaleza preventiva no sólo contribuye a la prevención de las consecuencias negativas relacionadas con la sexualidad, también puede mejorar la calidad de vida, la salud y el bienestar. De esta forma, la educación sexual favorece la promoción de la salud en general. (CENTRO FEDERAL DE SANIDAD, 2010, p.8).

Distintos autores/as apuntan lo siguiente sobre el significado de este término:

La educación afectivo-sexual es un proceso gradual que empieza en los primeros ańos de vida y se prolonga hasta la muerte. Debe partir de una concepción bio-psicosocial de la sexualidad, sin sobredimensionar ni excluir ninguna de estas tres variables, lo que permitirá múltiples perspectivas de trabajo y análisis, así como gozar de un fuerte componente transformador de la sociedad y de las concepciones de la misma, al analizar críticamente el concepto de sexualidad que se ha interiorizado y proponer alternativas más acordes con la felicidad y la calidad de vida de las personas. (CARRERA; LAMEIRAS; RODRÍGUEZ, 2007, p.192).

La EAS es hoy una demanda social basada en el derecho de los niños, niñas y adolescentes a ser informados sobre este tema. Esta información debe ser rigurosa, objetiva y completa a nivel biológico, psíquico y social, entendiendo la sexualidad como comunicación humana y fuente de salud, como afectividad y placer libre de los estereotipos de género. (FLECHA; NÚÑEZ, 2001, p.152).

La EAS no se ha venido transmitiendo en las escuelas (PELLEJERO; TORRES, 2011; VENEGAS, 2011a) es un término poco considerado en su totalidad por todos los profesionales de la educación que se encuentran impartiendo actualmente docencia. Por ello, se hace necesario revisar si durante las últimas décadas se han venido introduciendo en las leyes educativas algunos contenidos vinculados con lo afectivo - sexual. A continuación, abordamos esto último analizando detalladamente los preámbulos de las leyes educativas: LOGSE, LOCE, LOE y LOMCE. 
La educación afectivo-sexual en el sistema educativo español: análisis normativo y posibilidades de investigación

Revisando el preámbulo de la Ley Orgánica 1/1990, de 3 de octubre, de Ordenación General del Sistema Educativo (LOGSE), podemos observar, que la ley pretende introducir en el sistema educativo contenidos vinculados con la EAS:

El objetivo primero y fundamental de la educación es el de proporcionar a los niños y a las niñas, a los jóvenes de uno y otro sexo una formación plena que les permita conformar su propia y esencial identidad, así como construir una concepción de la realidad que integre a la vez el conocimiento y la valoración ética y moral de la misma. Tal formación plena ha de ir dirigida al desarrollo de su capacidad para ejercer, de manera crítica y en una sociedad axiológicamente plural, la libertad, la tolerancia y la solidaridad. (ESPAÑA, 1990, p.28927).

En la educación se transmiten y ejercitan los valores que hacen posible la vida en sociedad, singularmente el respeto a todos los derechos y libertades fundamentales, se adquieren los hábitos de convivencia democrática y de respeto mutuo, se prepara para la participación responsable en las distintas actividades e instancias sociales. La madurez de las sociedades se deriva, en muy buena medida, de su capacidad para integrar, a partir de la educación y con el concurso de la misma, las dimensiones individual y comunitaria. (ESPAÑA, 1990, p.28927).

La educación permite en fin, avanzar en la lucha contra la discriminación y la desigualdad, sean éstas por razón de nacimiento, raza, sexo, religión u opinión, tengan un origen familiar o social, se arrastren tradicionalmente o aparezcan continuamente con la dinámica de la sociedad. (ESPAÑA, 1990, p.28927).

Esos serán los fines que orientarán el sistema educativo español, de acuerdo con el Título Preliminar de esta ley y en el alcance de los mismos, la educación puede y debe convertirse en un elemento decisivo para la superación de los estereotipos sociales asimilados a la diferenciación por sexos, empezando por la propia construcción y uso del lenguaje. (ESPAÑA, 1990, p. 28928).

Se hace evidente, según visto en los párrafos anteriores, la necesidad de impartir una educación que permita a los alumnos-as formar su propia identidad, fomentando valores como la tolerancia y el respeto, que les permita la participación responsable en la sociedad. Asimismo se acentúa la importancia de una educación para todos-as, favoreciendo la igualdad y evitando la aparición de cualquier tipo de estereotipo de género.

En la misma línea sigue la Ley Orgánica 10/2002, de 23 de diciembre, de Calidad de la Educación (LOCE). Esta ley advierte la importancia de una 
educación de calidad para todos los alumnos-as (independientemente de su género) como oportunidad para conseguir un desarrollo óptimo tanto individual como social. Asimismo, expone la importancia de transmitir una serie de valores para respetar a los demás quedando reflejado en algunas de estos párrafos extraídos del preámbulo de la ley.

El logro de una educación de calidad para todos, que es el objetivo esencial de la presente ley, es un fin cuyas raíces se encuentran en los valores humanistas propios de nuestra tradición cultural europea. Y además, constituye, en el momento presente, un instrumento imprescindible para un mejor ejercicio de la libertad individual, para la realización personal, para el logro de cotas más elevadas de progreso social y económico y para conciliar, en fin, el bienestar individual y el bienestar social. (ESPAÑA, 2002, p.45188).

Tanto la Educación Infantil como la Educación Primaria se configuran como un período decisivo en la formación de la persona, ya que es en estas etapas cuando se asientan los fundamentos, no sólo para un sólido aprendizaje de las habilidades básicas en lengua, cálculo y lengua extranjera, sino que también se adquieren, para el resto de la vida, hábitos de trabajo, lectura, convivencia ordenada y respeto hacia los demás. En la Educación Primaria, además, se modifican la denominación de las áreas de conocimiento y los objetivos para conseguir una mejor adecuación a los fines que se pretenden. (ESPAÑA, 2002, p.45190).

Como hemos visto en las leyes anteriores, ya se observa una iniciación para impartir la EAS pero principalmente orientada hacia la igualdad de oportunidades y la prevención de desigualdades. Sin embargo, no es hasta la Ley Orgánica 2/2006, de 3 de mayo, de Educación (LOE) donde se hace una apuesta más notoria sobre contenidos relacionados con la EAS, notándose una mayor preocupación por la personalidad y el desarrollo de la afectividad de los alumnos y alumnas. Revisamos el preámbulo de la ley para señalar esta última afirmación:

La educación es el medio más adecuado para construir su personalidad, desarrollar al máximo sus capacidades, conformar su propia identidad personal y configurar su comprensión de la realidad, integrando la dimensión cognoscitiva, la afectiva y la axiológica. Para la sociedad, la educación es el medio de transmitir y, al mismo tiempo, de renovar la cultura y el acervo de conocimientos y valores que la sustentan, de extraer las máximas posibilidades de sus fuentes de riqueza, de fomentar la convivencia democrática y el respeto a las diferencias individuales, de promover la solidaridad y evitar la discriminación, con el objetivo fundamental de lograr la necesaria cohesión social. (ESPAÑA, 2006, p.1). 
La educación afectivo-sexual en el sistema educativo español: análisis normativo y posibilidades de investigación

Se trata de conseguir que todos los ciudadanos alcancen el máximo desarrollo posible de todas sus capacidades, individuales y sociales, intelectuales, culturales y emocionales para lo que necesitan recibir una educación de calidad adaptada a sus necesidades. (ESPAÑA, 2006, p.3).

En un lugar destacado aparece formulado el principio fundamental de la calidad de la educación para todo el alumnado, en condiciones de equidad y con garantía de igualdad de oportunidades. (ESPAÑA, 2006, p.7).

Entre los fines de la educación se resaltan el pleno desarrollo de la personalidad y de las capacidades afectivas del alumnado, la formación en el respeto de los derechos y libertades fundamentales y de la igualdad efectiva de oportunidades entre hombres y mujeres, el reconocimiento de la diversidad afectivo-sexual, así como la valoración crítica de las desigualdades, que permita superar los comportamientos sexistas. (ESPAÑA, 2006, p.7).

Finalmente, se hace referencia al alumnado extranjero, a las víctimas del terrorismo y de actos de violencia de género, al régimen de los datos personales de los alumnos, a la incorporación de créditos para la gratuidad del segundo ciclo de educación infantil y al fomento de la igualdad efectiva entre hombres y mujeres. (ESPAÑA, 2006, p.11).

Es necesario que la escuela favorezca el desarrollo de todas las dimensiones del alumno y la alumna, trabajando la afectividad y las emociones y fomentando la creación de vínculos que favorezcan las relaciones entre iguales que permita conocer mejor a los demás y a sí mismo, promoviendo la construcción de su propia identidad.

La Ley Orgánica 8/2013, de 9 de diciembre, para la Mejora de la Calidad Educativa (LOMCE), ley vigente en la actualidad, vuelve a insistir en la introducción dentro de los centros educativos de determinados valores como la libertad y la tolerancia para fomentar el respeto y la igualdad y conseguir una sociedad más justa. Así se especifica en el preámbulo:

La realidad familiar en general, y en particular en el ámbito de su relación con la educación, está experimentando profundos cambios. Son necesarios canales y hábitos que nos permitan restaurar el equilibrio y la fortaleza de las relaciones entre alumnos y alumnas, familias y escuelas. (ESPAÑA, 2013, p. 97859).

La recomendación del Comité de Ministros del Consejo de Europa a los Estados miembros sobre la Educación para la Ciudadanía Democrática, de fecha 16 de octubre de 2002, señala que la educación para la ciudadanía democrática es esencial para promover una sociedad libre, tolerante y justa y que contribuye a defender los valores y principios de la libertad, el pluralismo, 
los derechos humanos y el imperio de la ley, que son los fundamentos de la democracia (Ley 8/2013, de 20-12-2013, p. 97866).

Uno de los principios en los que se inspira el sistema educativo espańol es la transmisión y puesta en práctica de valores que favorezcan la libertad personal, la responsabilidad, la ciudadanía democrática, la solidaridad, la tolerancia, la igualdad, el respeto y la justicia, así como que ayuden a superar cualquier tipo de discriminación. Se contempla también como fin a cuya consecución se orienta el sistema educativo espańol la preparación para el ejercicio de la ciudadanía y para la participación activa en la vida económica, social y cultural, con actitud crítica y responsable y con capacidad de adaptación a las situaciones cambiantes de la sociedad del conocimiento. (ESPAÑA, 2013, p. 97866).

\section{Investigar en educación afectivo sexual}

La calidad educativa representa, hoy por hoy, uno de los objetivos prioritarios de cualquier sistema educativo. Se trata, en último término, de procurar las condiciones pedagógicas y sistémicas necesarias para ayudar a formar mejor a una ciudadanía más independiente y comprometida con la sociedad cambiante en la que viven. Para alcanzar estos fines es imprescindible no solo que la educación sino también que la investigación, constituyan procesos complementarios que aborden esta temática (JAKKU-SHIVONEN; NIEMI, 2011). La investigación representa, por tanto, un factor irrenunciable para cualquier docente. Autores como Stenhouse (1985) han defendido el papel del docente como investigador, de forma que la enseñanza sería investigación y los y las docentes investigadores en el aula y desde esta idea, sus acciones contribuirían al perfeccionamiento de la enseñanza. Autoras como Blanco (2010), conceden a la investigación un carácter fundamental para mejorar y enriquecer el perfil profesional de los y las docentes. Hablar de docentes investigadores es apostar por profesionales con saberes y competencias desarrolladas a propósito de la reflexión en su propia práctica.

Sin embargo, la formación inicial de los y las docentes españoles adolece de este carácter investigador. Pareciera que el maestro o maestra, lejos de ser un agente activo de cambio y trasformación, fuera un mero trasmisor de saberes, en un modelo que se antoja ya obsoleto. Un análisis de los planes de estudio y de las competencias exigidas a los futuros y futuras docentes españoles indica la ausencia de este perfil investigador, proponiéndose la necesidad de revisar esta situación y de al menos encontrar espacios formativos como los Trabajos Fin de Grado para iniciar e impulsar esta apuesta investigadora (BEJARANO; MATEOS; RODRIGUES, 2015). 
La educación afectivo-sexual en el sistema educativo español: análisis normativo y posibilidades de investigación

Un maestro que no investiga o que no puede hacerlo es un docente que quizás no pulse la realidad de su aula. Y la realidad indica que hay seres humanos sexuados que viven la sexualidad de diferente manera y que se sitúan fuera de lo que se ha venido a llamar la heteronormatividad (TRUJILLO, 2014). Para Hagger y Hazel (2006) es importante que los futuros y futuras docentes teoricen sobre la praxis, esto es, que reflexionen sobre sus propias formas de actuar. El y la docente deben mantener un diálogo reflexivo con la práctica de aula y con las situaciones relacionadas con la identidad sexual de las personas que acuden a ella. Schön (1987) denomina al proceso de dialogar con la práctica, reflexión en la acción y alude al profesional reflexivo. La falta de investigación normalizada en las Facultades de Educación y por extensión la ausencia de investigación centrada en la educación afectivo-sexual en las instituciones educativas (VENEGAS, 2011b) dificulta el que un maestro o maestra pueda abordar lo desconocido o lo que se pueda considerar "inusual". Un ejemplo de esto último, podría encontrarse en determinado alumnado de la etapa de infantil que manifiesta una identidad diferente a la de su propia sexualidad biológica. Como mantiene Butler (2005), la identidad de género no es expresiva del género anatómico sino que es construida socialmente. Ha habido un empeño por estabilizar la relación entre identidad de género, sexo y orientación sexual. Ello ha venido a reforzar socialmente a mujeres y varones heterosexuales, cuestionando otras identidades que no se someten a esa linealidad. El sistema educativo debe hacer posible, a través de la investigación, transformaciones epistemológicas que reconstruyan la lógica binaria heteronormativa y cuestionen sus efectos de jerarquía, clasificación, dominación y exclusión (LOURO, 2006).

Apostamos, por un modelo de maestro y maestra investigadora (BEJARANO; MATEOS; RODRIGUES, 2015) que a su vez trabaje desde una perspectiva critica de reconstrucción social. Este modelo mantiene que es necesario potenciar determinados valores en los agentes que se dedican a la educación para provocar un cambio social (PÉREZ GÓMEZ, 1992; LISTON; ZEICHNER, 1993; WELTMAN, 2000). Para Booth (2005) estos son valores sustantivos de la sociedad, enmarcados en lo que se entiende como una escuela inclusiva (VALCARCE, 2011), que para Thomazet (2009) está relacionada con elementos tan básicos como la equidad, la ética, la justicia social y en, último término, la democracia en su sentido más profundo.

\section{La necesidad de formar maestros y maestras en educación afectivo- sexual: propuesta de un instrumento de análisis curricular}

El estudio pormenorizado de las variables didácticas centradas en los libros de texto arroja dos tipos de sexismo en estos materiales: explícito y latente (PÉREZ, 2011). Si bien es cierto que la escuela ha sido una de las primeras 
instituciones en abandonar las prácticas segregadoras, también sabemos que en ella todavía se siguen reproduciendo actuaciones que inciden en la desigualdad entre sexos (BONAL, 2008). Frecuentemente, la escuela permanece inmovilista ante la descompensación personal, discursiva y de promoción socio-educativa de las niñas respecto a los niños. En la formación inicial de maestros y maestras se debe hacer una apuesta más formal por un paradigma inclusivo-crítico de reconstrucción social y democrática, como hemos apuntado anteriormente. Este cambio social tiene que surgir, como también hemos seńalado con anterioridad, a partir de una mejor formación investigadora del profesorado en temas de igualdad y diversidad afectivo-sexual para actuar y reflexionar sobre la práctica educativa. Esto posibilitará procesos de cuestionamiento en el propio currículum, en cuanto a los contenidos incluidos en él y en cuanto a su estructura (GIMENO, 2015). Se precisa un cambio de planteamiento institucional que dé paso a un currículum sexual (BEJARANO; MATEOS, 2014) que responda y esté a la altura de la realidad social.

Ante la necesidad de incorporar nuevas realidades afectivo-sexuales, exponemos una propuesta que consiste en el diseño de un instrumento de producción de datos relacionados con la EAS en materiales didácticos aplicados en las aulas de las etapas de Infantil y Primaria. Se trata de una tabla de análisis sobre contenidos relacionados con la Educación en igualdad y reconocimiento de las diversidades sexuales (Tabla 1 ). Dicha tabla ha sido elaborada en el contexto de la asignatura Trabajo Fin de Grado (año académico 2014-2015), por un grupo de trabajo formado por alumnado y profesorado participante de esta asignatura ubicada dentro del Grado de maestro de Educación Infantil y maestro de Educación Primaria. Se ha diseñado teniendo en cuenta la técnica de lectura reflexiva sobre distintas investigaciones llevadas a cabo por especialistas en la materia (BEJARANO; ORTIZ; VILLUENDAS, 2009; WEISS, 2001; MARTINEZ BARREIROS; 2004; AMEZUA, 1999; SÁNCHEZ SÁINZ, 2009; FALLAS; ARTAVIA; GAMBOA, 2012, etc.). La potencialidad de este instrumento de trabajo radica en la obtención de palabras claves relacionadas con la EAS. Estas palabras, también llamadas categorías, se han definido y ordenado con el objetivo que se identifiquen a partir de ellas contenidos curriculares afines a lo afectivo-sexual en libros de texto, guías docentes y libros de lectura.

La finalidad de este instrumento es realizar análisis documentales referidos a la EAS en los materiales curriculares utilizados en el aula de infantil y primaria. El análisis documental es una técnica de registro de gran utilidad para la obtención de información retrospectiva y referencial sobre un fenómeno o un programa concreto, en este caso la EAS. Dicha tabla de análisis facilitará la extracción de datos de manera sistemática y planificada sobre documentos 
La educación afectivo-sexual en el sistema educativo español: análisis normativo y posibilidades de investigación

ya escritos que abarcan una amplia gama de modalidades didácticas. Los documentos son una fuente fidedigna y práctica para revelar los intereses y las perspectivas de quienes los han diseñado, pudiendo proporcionar información valiosa a la que quizás no se tenga acceso a través de otros medios.

Tabla 1 - Instrumento de análisis.

Tabla de análisis (Nombre del documento que está registrando) Nivel educativo:

\begin{tabular}{|c|c|}
\hline PALABRAS CLAVES/Categorías & \multirow[t]{15}{*}{ Observaciones. } \\
\hline Educar en Igualdad & \\
\hline Educar en la Tolerancia & \\
\hline Educar en la Salud & \\
\hline Relaciones afectivo-sexuales & \\
\hline Cuerpo: cambios corporales, etc. & \\
\hline Sistema sexo-género & \\
\hline Identidades sexuales & \\
\hline Abuso/Violencia sexual & \\
\hline Diversidad sexual & \\
\hline Prácticas sexuales & \\
\hline Fobias sexuales (homofobia, transfobia, etc.) & \\
\hline Afectividad/Placer/Ternura,etc. & \\
\hline Sexualidad /Educación sexual & \\
\hline Diversidad Familiar & \\
\hline
\end{tabular}

Fuente: Elaboración propia.

Las instituciones escolares producen y reproducen discursos discriminatorios pero como recuerda Torres (2008), en la medida que políticamente se les encargan educar, deben desempeñar un papel mucho más activo como espacios de resistencia y de denuncia de discursos y prácticas que hoy, dentro de sus aulas, siguen originando marginación.

Siguiendo con este argumento, Pellejero y Torres (2011) indican que podemos intuir que en la mayoría de los casos el currículo oculto continúa reproduciendo modelos de discriminación aunque en el currículo explícito encontremos aspectos que denotan una cierta tendencia a la igualdad. Es necesario pues, rastrear qué conocimientos sobre la diversidad sexual se ofrecen en los materiales que explicitan el currículum oficial y que son 
utilizados a diario en las aulas, concretamente en los libros de texto. El libro de texto, es un mediador curricular básico además de un instrumento cultural y socializador y como tal mediador persigue de forma manifiesta, latente $\mathrm{u}$ oculta, no solo transmitir información escolar sino también que el alumnado adquiera las normas, valores e ideologías predominantes de la sociedad en la que se desenvuelven e incorpore otros modelos sexuales cada vez más visibilizados.

\section{Consideraciones Finales}

Las cuestiones de sexualidad y género representan un objetivo social que no podemos eludir. Sin embargo, las demandas sociales no son habitualmente acompañadas por avances legislativos. Si se analizan las leyes españolas desde la llegada de la democracia, se han producido tímidos progresos en materia de sexualidad e igualdad de género que no responden a la realidad emergente donde la diversidad afectivo-sexual es cada vez más explícita (SOARES; TOLEDO, 2012; BEJARANO; MATEOS, 2014). En ese escenario es comprensible que los materiales educativos de cualquier etapa no recojan de forma significativa los asuntos relacionados con la diversidad sexual, afectiva y la libertad individual (GÓMARA; et al., 2010) como reflejo de la propia ausencia o ambigüedad legislativa. Frente a esta situación parece necesario reflexionar sobre qué papel queremos cumpla la institución escolar, si debe ser ajena a la evolución de la propia ciudadanía o adaptarse para trabajar y dar cabida a las diferentes dimensiones afectivas y sexuales.

Como hemos mostrado en este artículo, el análisis de los preámbulos de las distintas leyes educativas españolas de las últimas décadas deja clara evidencia de la escasa vocación que existe en el sistema educativo español por un tipo de educación, como es la afectivo-sexual, que entronca directamente con un aspecto íntimo de la personalidad humana. Los profesionales de la educación, ya sean formadores de formadores, educadores sociales o maestros y maestras han de asumir el reto de la diversidad social y esto significa, entre otras cosas, aceptar la responsabilidad de contribuir a la formación de ciudadanos y ciudadanas que contribuyan a seguir avanzando en la construcción de un mundo diverso. Para ello, todas las propuestas curriculares y los diseños de enfoque investigador deben asumir esta parte de la dimensión humana, la diversidad afectivo-sexual, pero de manera integral. Las aulas han de recibir profesionales de la docencia que ayuden a que cada niño y niña construya la mejor imagen de sí mismo y del otro-a y a reforzar las plenas garantías en el ejercicio del derecho a ser y estar en la sociedad de manera libre y sin que ello reporte discriminación e invisibilidad. 
La educación afectivo-sexual en el sistema educativo español: análisis normativo y posibilidades de investigación

\section{REFERENCIAS}

AMEZUA, E. Teoría de los sexos. Revista Española de Sexología, Madrid, n. 95-96, p.1223, 1999.

BEJARANO, M.; MATEOS, A. Género y sexualidad en la formación inicial de maestros y maestras. ¿Por qué no un currículum sexual? Exedra, Suplemento Sexualidade, Género e Educaçáo, [Coimbra], p.127-146, dez. 2014. Edición temática.

BEJARANO, M.; MATEOS, A.; RODRIGUES, A. Los trabajos fin de grado en la formación inicial de los y las docentes: hacia un modelo basado en la competencia investigadora. In: RODRÍGUEZ TORRES, J. (Coord.). Experiencias de adaptación en el EEES. Madrid: McGraw-Hill, 2015. p.121-134.

BEJARANO, M.; ORTIZ, C.; VILLUENDAS, D. Sexualidad y género: la sexualidad y educación en la mujer. Transatlántica de Educación, Mexico, n.6, p.67-78, 2009.

BEJARANO, M.; RODRÍGUEZ TORRES, J. La formación pedagógica de futuros maestros y maestras: otros ámbitos para la actuación. In: MATEOS, A.; MANZANARES, A. (Dir.). Mejores maestros, mejores educadores. Málaga: Aljibe, 2015. p.43-61.

BLANCO, N. La investigación en el ámbito el currículum y como método para su desarrollo. In: SACRISTÁN GIMENO, J. (Comp.). Saberes e incertidumbres sobre el currículum. Madrid: Morata, 2010. p. 569-584.

BONAL, X. Las actitudes del profesorado ante la coeducación: propuestas de intervención. Barcelona: Graó, 2008.

BOOTH, T. Keeping the future alive: putting inclusive values in action. Forum, Reino Unido, v. 47, n 2-3, p. 151-158, 2005.

BUTLER, J. Cuerpos que importan. Buenos Aires: Paidós, 2005.

CARRERA, M. V.; LAMEIRAS M.; RODRÍGUEZ, Y. Intervención y evaluación de un programa de educación afectivo-sexual en la escuela para padres y madres de adolescentes. Revista Diversitas, Bogotá, v. 3, n. 2, p. 191-202. 2007. Disponible en: <http://www.usta. edu.co/otraspaginas/diversitas/doc_pdf/diversitas_6/vol.3no.2/articulo_1.pdf>. Aceso en: 20 dic. 2014.

CENTRO FEDERAL DE SANIDAD, EDUCACIÓN OFICINA REGIONAL DE LA OMS PARA EUROPA. Estándares de educación sexual en Europa. Madrid: [s.n.], 2010. Disponible en: <http://www.madridsalud.es/publicaciones/OtrasPublicaciones/standars_de_ calidad_de_la_educacion_sexual_en_europa_traducido_12nov.pdf $>$. Aceso en: 10 dic. 2014.

CEVAllos, A. C. ¿Educación sexual para mi hijo e hija de preescolar (3-5 años)? percepciones de padres y madres de familia. Revista Electrónica Educare, Costa Rica, v. 18, n. 3, p. 91-110, 2014.

ESPAÑA. Ley orgánica 8/2013, de 9 de diciembre de 2013. Para la mejora de la calidad educativa. Boletín Oficial del Estado, España, n. 295, p. 97858, dic. 2013.

Ley orgánica 2/2006, de 3 de mayo de 2006. Ley orgánica de calidad de la educación. Boletín Oficial del Estado, España, n.106, p. 17158, may. 2006. 
. Ley orgánica 1/2004, de 28 de diciembre de 2004. Medidas de protección integral contra la violencia de género. Boletín Oficial del Estado, España, n. 313, p. 42166, dic. 2004.

. Ley orgánica 10/2002, de 23 de diciembre de 2002. Calidad de la educación. Boletín Oficial del Estado, España, n. 307, p. 45188, dic. 2002.

Ley orgánica 1/1990, de 3 de octubre de 1990. Ordenación general del sistema educativo. Boletín Oficial del Estado, España, n. 238, p. 28927, out. 1990.

FALLAS, M. A.; ARTAVIA, C.; GAMBOA, A. Educación sexual: orientadores y orientadoras desde el modelo biográfico y profesional. Revista electrónica Educare, Costa Rica, n. 16, p. 53-71, 2012.

FLECHA, C.; NÚNEEZ, M. (Ed.). La educación de las mujeres: nuevas perspectivas. Sevilla: Universidad de Sevilla, 2001. p. 147-160.

GÓMARA, I.; REPARAZ, C. H.; OSORIO, A.; DE IRALA, J. La educación sexual en los textos escolares españoles: evaluación de un texto alternativo. Estudios sobre Educación, Navarra, v. 18, p. 139-164, 2010.

GIMENO, J. (Comp.). Los contenidos, una reflexión necesaria. Madrid: Morata, 2015.

JAKKU-SIHVONENE; NIEMI, H. (Ed.). Aprender de Finlandia: la apuesta por un profesorado investigador. Madrid: Ministerio de Educación, Cultura y Deporte y Kaleida Forma, 2011.

LISTON, D. P. Y.; ZEICHNER, K. M. Formación del profesorado y condiciones sociales de escolarización. Madrid: Morata, 1993.

LOURO, G. Um corpo estranho, ensaios sobre sexualidade e teoria queer. Revista Crítica de Ciências Sociais, Coimbra, n. 76, p. 145-149, 2006.

MALÓN, A. ¿El derecho a una educación sexual? Entre los discursos de salvación y la ausencia del conocimiento. Educatio Siglo XXI, Murcia, v. 30, n.2, p. 207-228, 2012.

MARTÍNEZ BARREIROS, A. La construcción social del cuerpo en las sociedades contemporáneas. Papers: Revista de Sociologia, Bellaterra, n. 73, p. 127-152, 2004.

PELLEJERO, L.; TORRES, B. La educación de la sexualidad, el sexo y el género en los libros de texto de educación primaria. Revista de Educación, Madrid, n.354, p. 399-427, 2011.

PENNA, M. Formación del profesorado en la atención a la diversidad afectivosexual. 2012. 670f. Tesis (Doctoral en Educacion) - Facultad de Educación, Universidad Complutense de Madrid, Madrid, 2012. Disponible en: <http://eprints.ucm.es/16718/1/ T34011.pdf>. Acceso en: 10 jul. 2015.

PÉREZ, A. El sexismo en los manuales escolares de ciencias sociales de la E.S.O. 2012. 335f. Dissertacion (Mestrado em Pesquisa e Avaliação) - Departamento de Didáctica y Organización Escolar, Universidad De Almería, Almeira, 2012. Disponible en: <http:// repositorio.ual.es/jspui/handle/10835/1142\#.UTIgBTcdX6s>. Aceso en: 26 nov. 2015.

PÉREZ GÓMEZ, A. La función y formación del profesor/a en la enseñanza para la comprensión: diferentes perspectivas. In: GIMENO SACRISTÁN, J.; PÉREZ GÓMEZ A. I. (Ed.). Comprender y trasformar la enseńanza. Madrid: Morata, 1992. p. 76-89.3 
La educación afectivo-sexual en el sistema educativo español: análisis normativo y posibilidades de investigación

SÁNCHEZ SÁINZ, M. (Coord.). Cómo educar en la diversidad en la diversidad afectiva, sexual y personal en educación infantil: orientaciones prácticas. Madrid: La Catarata, 2009.

SOARES, M.; DE TOLEDO, M. Dialogando con estudios acerca de las vivencias afectivosexuales de las parejas de transexuales. Liberabit, Lima, v. 18, n. 2. p. 25-29, 2012.

SCHÖN, D. La formación de profesionales reflexivos: hacia un nuevo diseño de la enseñanza y el aprendizaje de las profesiones. Barcelona: Paidós, 1987. p. 38-51.

STENHOUSE, L. El profesor como tema de investigación y desarrollo. Revista de Educación, Madrid, n. 277, p. 43-53. 1985.

THOMAZET, S. From integration to inclusive education: does changing the terms improve practice? International Journal of Inclusive Education, Reino Unido, v. 13, n. 6, p. 553563, 2009.

TORRES, J. Diversidad cultural y contenidos escolares. Revista de Educación, Madrid, n.345, p.83-110, 2008.

TRUJILLO, G. De la necesidad y urgencia de seguir queerizando y trans-formando el feminismo. Unas notas para el debate desde el contacto español: ex aequo. Revista da Associaçáo Portuguesa de Estudios sobre as Mulheres, Portugal, n. 29, p. 55-68, 2014.

VALCARCE, M. De la escuela integradora a la escuela inclusiva. Innovación Educativa, Santiago de Compostela, n. 21, p. 119-131, 2011.

VENEGAS, M. El modelo actual de educación afectivo sexual en España: el caso de Andalucía. Revista Iberoamericana de Educación, Madrid, n. 55, p.1-10, 2011a.

La investigación acción educativa en educación afectivosexual: una metodología para el cambio social. EMPIRIA: Revista de Metodología de Ciencias Sociales, Madrid, n. 21, p. 39-61, 2011b.

WEISS, M. Educación sexual infantil/juvenil. Revista Ensayos y Experiencias, [S.1.], n. 38, p. 211-232, 2001.

WELTMAN, B. D. Reconsidering Arthur Bestor and the cold war in social education. Theory and Research in Social Education, [S.1.], n.28, p.11-39, 2000. 


\section{Resumen}

La educación afectivo-sexual en el sistema educativo español: análisis normativo y posibilidades de investigación

En este artículo se realiza un análisis de los preámbulos de las leyes educativas aprobadas en Espańa desde 1990 hasta 2013 con el objetivo de demostrar la presencia/ausencia de contenidos relacionados con la educación afectivo-sexual. Ello nos permitirá comprobar la importancia que se le ha venido otorgando a esta dimensión humana y educativa en la escuela desde finales del siglo XIX hasta la actualidad. Asimismo, profundizamos en la relevancia de dos aspectos para la formación de maestros y maestras. El primero el abordar temáticas educativas sobre igualdad y el segundo adquirir habilidades en investigación sobre educación afectivo-sexual en la práctica docente. Ambas cuestiones fomentarán un perfil docente reflexivo-práctico. Por último, se propone un instrumento de análisis curricular como recurso de producción de datos relacionados con la temática abordada.

Palabras Clave: Educación afectivo-sexual. Sistema educativo. Leyes educativas. Investigación educativa.

\section{Abstract \\ The affective-sexual education in the spanish education system: normative analysis and research possibilities}

This paper presents an analysis of the preambles of the education laws adopted in Spain from 1990 to 2013 with the aim of demonstrating the presence/absence of the contents related to the affective sexual education. It will let us test the importance that has been given to this subject of the human personality from de educative field at school since the late nineteenth century to the present. Additionally, we will delve into the importance of two aspects for training teachers. The first one would address educational issues about equality and the second one would acquire research skills about affective-sexual education in the teaching practice. Both issues will encourage a reflexive-practice teacher profile. In the end, it is proposed a curricular analysis tool as a resource to produce datum concerning the addressed subject.

Keywords: Affective-sexual education. Education system. Education laws. Educative research. 
